hep-th/0604111

April 2006

\title{
Master Higher-Spin Particle
}

\author{
Sergey Fedoruk ${ }^{\star}$, Evgeny Ivanov \\ Bogoliubov Laboratory of Theoretical Physics, JINR, \\ 141980 Dubna, Moscow region, Russia \\ fedoruk, eivanov@theor.jinr.ru
}

\begin{abstract}
We propose a "master" higher-spin (HS) particle system. The particle model relevant to the unfolded formulation of HS theory, as well as the HS particle model with a bosonic counterpart of supersymmetry, follow from the master model as its two different gauges. Quantization of the master system gives rise to a new form of the massless HS equations in an extended space involving, besides extra spinorial coordinates, also a complex scalar one. As solutions to these equations we recover the massless HS multiplet with fields of all integer and half-integer helicities, and obtain new multiplets with a non-zero minimal helicity. The HS multiplets are described by complex wave functions which are holomorphic in the scalar coordinate and carry an extra $U(1)$ charge $q$. The latter fully characterizes the given multiplet by fixing the minimal helicity as $q / 2$. We construct a twistorial formulation of the master system and present the general solution of the associate HS equations through an unconstrained twistor "prepotential".
\end{abstract}

PACS: 11.30.Ly, 11.30.Pb, 11.10.Ef

Keywords: Higher spins, twistors, supersymmetry

${ }^{\star}$ On leave from Ukrainian Engineering-Pedagogical Academy, Kharkov, Ukraine 


\section{Introduction}

The latest developments in higher spin (HS) theory clearly show the importance of (super)spaces with extra bosonic co-ordinates for concise and suggestive formulations of this theory (see e.g. [1]-[9]). The simple and, at the same time, powerful device to analyze the geometric structure of such (super)spaces is (super)particles propagating in them. For instance, the free HS equations in different formulations can be reproduced as a result of first quantization of the appropriate (super)particle $[10,11,5]$.

The model of tensorial superparticle $[10,11]^{1}$ provides a world-line interpretation of the unfolded formulation of the HS superfield theory [5]. Actually, the latter can be equivalently given either in a hyperspace containing a ten-dimensional bosonic subspace alongside an extra commuting Weyl spinor, or in superspace with the Grassmann spinor coordinate (quantization of tensorial superparticle and links of it to an unfolded formulation were also studied in $[6,13,14$, 15]). The ten-dimensional bosonic subspace is parameterized by a position four-vector $x^{\alpha \dot{\beta}}$ (it constitutes Minkowski subspace) and six tensorial coordinates $z^{\alpha \beta}, \bar{z}^{\dot{\alpha} \dot{\beta}}$. The crucial advantage of using this ten-dimensional space-time is that it manifests the underlying $O S p(1 \mid 8)$ covariance of the unfolded formulation.

There also exists a version of the unfolded formulation which makes no use of the tensorial coordinates $[5,6,15]$ (the construction of HS conformal currents in such a formulation was a subject of recent paper [16]). This version is more economic, although the $S p(8)$ (and $O S p(1 \mid 8)$ ) symmetry is hidden in its framework. In the pure bosonic case the basic equation for the HS field $\Phi(y, \bar{y}, x)[5]$ reads

$$
\left(\partial_{\alpha \dot{\alpha}}+i \frac{\partial}{\partial y^{\alpha}} \frac{\partial}{\partial \bar{y}^{\dot{\alpha}}}\right) \Phi=0,
$$

where $y^{\alpha}$ is a commuting Weyl spinor, $\bar{y}^{\dot{\alpha}}=\overline{\left(y^{\alpha}\right)}$. Solution of the unfolded equation (1.1) can be found, assuming the polynomial dependence of the wave function on the commuting spinors $y^{\alpha}, \bar{y}^{\dot{\alpha}}$

$$
\Phi(x, y, \bar{y})=\sum_{m=0}^{\infty} \sum_{n=0}^{\infty} y^{\alpha_{1}} \ldots y^{\alpha_{m}} \bar{y}^{\dot{\alpha}_{1}} \ldots \bar{y}^{\dot{\alpha}_{n}} \varphi_{\alpha_{1} \ldots \alpha_{m} \dot{\alpha}_{1} \ldots \dot{\alpha}_{n}}(x) .
$$

Independent space-time fields in the expansion of the general field (1.2) are self-dual $\varphi_{\alpha_{1} \ldots \alpha_{m}}$ and anti-self-dual $\varphi_{\dot{\alpha}_{1} \ldots \dot{\alpha}_{n}}$ field strengths of all helicities (including the half-integer ones) $[15,16]$. All other fields are expressed as $x$-derivatives of these basic ones. A classical counterpart of this formulation is the particle system with the action [5]

$$
S_{1}^{H S}=\int d \tau\left(\lambda_{\alpha} \bar{\lambda}_{\dot{\alpha}} \dot{x}^{\dot{\alpha} \alpha}+\lambda_{\alpha} \dot{y}^{\alpha}+\bar{\lambda}_{\dot{\alpha}} \dot{\bar{y}}^{\dot{\alpha}}\right) .
$$

The spinors $\lambda_{\alpha}, \bar{\lambda}_{\dot{\alpha}}$ are canonical momenta for $y^{\alpha}, \bar{y}^{\dot{\alpha}}$, "dot" on fields denotes the time derivative. The first class constraints

$$
P_{\alpha \dot{\alpha}}-\lambda_{\alpha} \bar{\lambda}_{\dot{\alpha}} \approx 0
$$

after quantization reproduce the unfolded equation (1.1).

A different model of the massless HS particle was proposed in [17]. A distinguishing feature of this model is its manifest covariance under the even counterpart of $4 D$ supersymmetry ${ }^{2}$.

\footnotetext{
${ }^{1}$ See $[12]$ for the further related developments.

${ }^{2}$ It is worth mentioning that a model with the broken even "supersymmetry" was used in $[18,19]$ to describe a particle with fixed spin. The even "supersymmetry" was also exploited in [20] for the description of spectrum of the critical open $N=2$ string in $2+2$ dimensions.
} 
The action resembles that of the usual massless $N=1$ superparticle

$$
\begin{gathered}
S_{2}^{H S}=\int d \tau\left(P_{\alpha \dot{\alpha}} \omega^{\dot{\alpha} \alpha}-e P_{\alpha \dot{\alpha}} P^{\alpha \dot{\alpha}}\right), \\
\omega^{\dot{\alpha} \alpha} \equiv \dot{x}^{\dot{\alpha} \alpha}-i \bar{\zeta}^{\dot{\alpha}} \dot{\zeta}^{\alpha}+i \dot{\bar{\zeta}}^{\dot{\alpha}} \zeta^{\alpha} .
\end{gathered}
$$

However, the crucial difference from the superparticle case is that the Weyl spinor $\zeta^{\alpha}, \bar{\zeta}^{\dot{\alpha}}=\left(\overline{\zeta^{\alpha}}\right)$, is commuting. The set of the Hamiltonian constraints in the system includes the mass-shell constraint $P_{\alpha \dot{\alpha}} P^{\alpha \dot{\alpha}} \approx 0$ and the even spinor constraints

$$
D_{\alpha} \equiv \pi_{\alpha}+i P_{\alpha \dot{\alpha}} \bar{\zeta}^{\dot{\alpha}} \approx 0, \quad \bar{D}_{\dot{\alpha}} \equiv \bar{\pi}_{\dot{\alpha}}-i \zeta^{\alpha} P_{\alpha \dot{\alpha}} \approx 0
$$

where $\pi_{\alpha}$ and $\bar{\pi}_{\dot{\alpha}}$ are conjugate momenta for $\zeta^{\alpha}$ and $\bar{\zeta}^{\dot{\alpha}}$. The spinor constraints (1.7) have the Poisson brackets algebra

$$
\left[D_{\alpha}, \bar{D}_{\dot{\alpha}}\right]_{P}=2 i P_{\alpha \dot{\alpha}}
$$

which constitutes a classical version of the even "supersymmetry" algebra of covariant derivatives with commutators instead of anticommutators. Half of the spinor constraints (1.7) is first class whereas another half is second class. Quantization of the system (1.5) by Gupta-Bleuler (or analytic) method ${ }^{3}$ was performed in [17]. The wave function of the even "supersymmetry" particle obtained as a result of this quantization procedure is an even counterpart of chiral $N=1$ superfield

$$
\Psi\left(x_{L}, \zeta\right)=\sum_{n=0}^{\infty} \zeta^{\alpha_{1}} \ldots \zeta^{\alpha_{n}} \psi_{\alpha_{1} \ldots \alpha_{n}}\left(x_{L}\right)
$$

depending on $x_{L}^{\dot{\alpha} \alpha}=x^{\dot{\alpha} \alpha}+i \bar{\zeta}^{\dot{\alpha}} \zeta^{\alpha}$ and $\zeta^{\alpha}$. Besides the chirality condition

$$
\bar{D}_{\dot{\alpha}} \Psi=0 \text {, }
$$

this field is subjected to the equations

$$
\partial_{L}^{\dot{\alpha} \alpha} \partial_{\alpha} \Psi=0, \quad \partial_{L}^{\dot{\alpha} \alpha} \partial_{L} \alpha \dot{\alpha} \Psi=0,
$$

which are quantum counterparts of first class constraints. Due to eqs. (1.11) the fields in the expansion of the wave function with respect to the even spinor variables are self-dual field strengths of the massless particles of all helicities. Anti-self-dual field strengths are contained in the complex conjugated (anti-chiral) field.

The aim of this paper is to formulate and study a new ("master") model of HS particle which yields both models (1.3) and (1.5) upon choosing the appropriate gauges and, after quantization, both systems of the HS equations (1.1) and (1.10), (1.11) as the result of partial solving of the full set of relevant equations. One of the novel features of this system is that, instead of the set (1.1), the master model actually implies a modified unfolded system of equations in which the infinite towers of higher spins are accommodated by some holomorphic functions depending on a new scalar complex variable. These functions are characterized by the "external" $U(1)$ charge number $q$ which fully specifies the corresponding infinite-dimensional multiplet of spins and has, as its classical counterpart, the coefficient before Fayet-Iliopoulos term in the "master" action. The HS multiplets can start not only with a scalar field (as in (1.2)), which corresponds

\footnotetext{
${ }^{3}$ In application to superparticle models, this method was pioneered in [21].
} 
to the choice $q=0$, but also with any other self-dual field the helicity of which is fixed as $q / 2$ by the external $U(1)$ charge.

In Sect. 2 we give a general description of our model. The master HS particle propagates in the space parameterized by the spinorial variables of the systems (1.3), (1.5) and an additional complex scalar. The Hamiltonian formulation of this system involves only first class constraints. They include unfolded constraints (1.4), first class generalization of the spinor constraints (1.7) and a scalar constraint which generates local $U(1)$ transformations of the twistor-like variables and complex scalar co-ordinate.

In Sect. 3 we carry out canonical quantization of the master system and obtain the relevant set of HS equations for the wave function. This set generalizes and involves both sets (1.1) and $(1.10),(1.11)$. Notably, the unfolded equation similar to (1.1) proves to be a consequence of the quantum counterparts of the spinor constraints. There is also a scalar $U(1)$ constraint which is an analog of the "spin-shell" constraint present in the model of massless particle with fixed helicity [22]-[25]. In our case the degree of homogeneity of the HS field with respect to commuting twistor-like spinors is not fixed due to the presence of complex scalar coordinate $\eta$ with non-zero $U(1)$ charge. The external $U(1)$ charge $q$ of the HS wave function in extended space is defined as a degree of homogeneity with respect to both spinor and scalar co-ordinates. The wave function is holomorphic in $\eta$. At any fixed $q$, there is an infinite set of states in the spectrum.

At $q=0$ the HS wave function describes massless particles of all helicities in terms of their complex self-dual field strengths (starting with a complex scalar field). The $q=0$ HS field can be treated as a holomorphic "half" of the Vasiliev's unfolded field (1.2). The latter is recovered as a sum of the original HS field (holomorphic in $\eta$ ) and its complex conjugate (antiholomorphic in $\eta$ ) which comprises anti-self-dual field strengths. At $q \neq 0$ we obtain new HS multiplets having different helicity contents. Physical states of the HS fields with $q>0$ are massless particles with the helicities ranging from $\frac{q}{2}$ to infinity. When $q<0$, the spectrum contains an infinite tower of massless states of all positive helicities like in the $q=0$ case and, additionally, the finite number of states with negative helicities described by anti-self-dual field strengths (such HS multiplets can be named "spin-flip" ones).

All these HS multiplets admit an equivalent (and more economic) description in terms of the chiral and anti-chiral fields of the bosonic "supersymmetry". At $q=0$ it is just the HS field (1.9). The $q \neq 0$ multiplets are described by the properly constrained chiral fields with external indices.

We also construct a twistor formulation of the master system. In contrast to the twistor formulation of the system (1.3), the master HS particle propagates in a space parametrized by a unit twistor and an additional complex scalar. We construct a coordinate twistor transform relating different classical formulations of the master system, as well as a field twistor transform which allows one to reconstruct the space-time HS fields by the twistorial "prepotential" which solves the HS equations.

In Sect. 4 we summarize our results and make some comments on the symmetries of the master HS particle. 


\section{Master HS particle model}

\subsection{Action and constraints}

In this section we describe a new model of even HS particle effectively possessing only first class constraints. It plays the role of the "master system" both for the particle model (1.3) corresponding to the unfolded formulation and for the model (1.5) with the explicit even "supersymmetry".

The master system involves the variables of both systems (1.3) and (1.5) and also an additional complex scalar $\eta(\bar{\eta}=(\bar{\eta}))$. The model is described by the following action

$$
S_{m a s t}^{H S}=\int d \tau\left[\lambda_{\alpha} \bar{\lambda}_{\dot{\alpha}} \omega^{\dot{\alpha} \alpha}+\lambda_{\alpha} \dot{y}^{\alpha}+\bar{\lambda}_{\dot{\alpha}} \dot{\bar{y}}^{\dot{\alpha}}+i(\eta \dot{\bar{\eta}}-\dot{\eta} \bar{\eta})-2 i \bar{\eta} \dot{\zeta}^{\alpha} \lambda_{\alpha}+2 i \eta \bar{\lambda}_{\dot{\alpha}} \dot{\bar{\zeta}}^{\dot{\alpha}}-l(\mathcal{N}-c)\right] .
$$

The action is invariant under local phase transformations of the involved complex fields except the fields $\zeta, \bar{\zeta}$, with

$$
\mathcal{N} \equiv i\left(y^{\alpha} \lambda_{\alpha}-\bar{\lambda}_{\dot{\alpha}} \bar{y}^{\dot{\alpha}}\right)-2 \eta \bar{\eta}
$$

being the $U(1)$ current, $l$ the relevant gauge field and $c$ a strength of the $1 D$ "Fayet-Iliopoulos" term. The field $l$ acts as a Lagrange multiplier effecting the constraint

$$
i\left(y^{\alpha} \lambda_{\alpha}-\bar{\lambda}_{\dot{\alpha}} \bar{y}^{\dot{\alpha}}\right)-2 \eta \bar{\eta}-c \approx 0
$$

which generates, in the Hamiltonian formalism, local $U(1)$ transformations. This constraint is the HS generalization of the "spin-shell" constraint $i\left(y^{\alpha} \lambda_{\alpha}-\bar{\lambda}_{\dot{\alpha}} \bar{y}^{\dot{\alpha}}\right)-c \approx 0$ present in the model of massless particle with a fixed helicity [22]-[25].

Hamiltonian analysis of the system (2.1) is performed in Appendix A. Besides the constraint (2.3), the action (2.1) also gives rise to the following set of the primary constraints

$$
\begin{gathered}
T_{\alpha \dot{\alpha}} \equiv P_{\alpha \dot{\alpha}}-\lambda_{\alpha} \bar{\lambda}_{\dot{\alpha}} \approx 0, \\
\mathcal{D}_{\alpha} \equiv D_{\alpha}+2 i \bar{\eta} \lambda_{\alpha} \approx 0, \quad \overline{\mathcal{D}}_{\dot{\alpha}} \equiv \bar{D}_{\dot{\alpha}}-2 i \eta \bar{\lambda}_{\dot{\alpha}} \approx 0,
\end{gathered}
$$

where $D_{\alpha}$ and $\bar{D}_{\dot{\alpha}}$ are defined in (1.7). Note that, by the same token as in [22]-[25], the second class constraints

$$
p_{\eta}+i \bar{\eta} \approx 0, \quad \bar{p}_{\eta}-i \eta \approx 0
$$

also following from (2.1) can be treated in the strong sense by introducing Dirac brackets for them. Then the complex scalar $\eta$ and its complex conjugate form the canonical pair

$$
[\eta, \bar{\eta}]_{D}=\frac{i}{2}
$$

The canonical brackets for another phase variables do not change

$$
\begin{gathered}
{\left[x^{\dot{\alpha} \alpha}, P_{\beta \dot{\beta}}\right]_{D}=\delta_{\beta}^{\alpha} \delta_{\dot{\beta}}^{\dot{\alpha}}, \quad\left[\zeta^{\alpha}, \pi_{\beta}\right]_{D}=\delta_{\beta}^{\alpha}, \quad\left[\bar{\zeta}^{\dot{\alpha}}, \bar{\pi}_{\dot{\beta}}\right]_{D}=\delta_{\dot{\beta}}^{\dot{\alpha}},} \\
{\left[y^{\alpha}, \lambda_{\beta}\right]_{D}=\delta_{\beta}^{\alpha}, \quad\left[\bar{y}^{\dot{\alpha}}, \bar{\lambda}_{\dot{\beta}}\right]_{D}=\delta_{\dot{\beta}}^{\dot{\alpha}} .}
\end{gathered}
$$

The only non-vanishing bracket of the constraints $(2.3)-(2.5)$ is

$$
\left[\mathcal{D}_{\alpha}, \overline{\mathcal{D}}_{\dot{\alpha}}\right]_{D}=2 i T_{\alpha \dot{\alpha}}
$$


Therefore, all constraints (2.3)-(2.5) are effectively first class and the quantization procedure is straightforward.

Note that one can avoid passing to Dirac brackets for the second class constraints (2.6) and quantize the whole system by Gupta-Bleuler (or analytic) method. As shown in Appendix A, in this approach one obtains the same set of equations on the wave function.

Let us now explain in which way the master system is gauge-equivalent to the HS particle systems (1.5) and (1.3).

The systems (1.5) and (2.1) are (classically) equivalent to each other in the common sector of their phase space. This sector is singled out by choosing the definite sign of the energy $P_{0}$ which is fixed due to the constraint (2.4). This equivalence becomes evident if one observes that the system (2.1) can be interpreted as the system (1.5) in which the second class constraints are converted into first class by introducing new canonical pair $\eta, \bar{\eta}$.

To be more precise, we use the covariant conversion method firstly proposed in [23] for the case of usual superparticle. To convert two second class constraints contained in the spinor constraints (1.7) into first class, we introduce two additional degrees of freedom carried by the complex scalar field $\eta$. We also introduce a commuting Weyl spinor $\lambda_{\alpha}$ to ensure the Lorentz covariance of the new spinor constraints (2.5). The closure of the algebra (2.10) of new spinor constraints gives just the constraint (2.4) resolving four-momentum in terms of the spinor product. This resolution is defined up to an arbitrary phase transformation of $\lambda_{\alpha} \bar{\lambda}_{\dot{\alpha}}$. In order to ensure this $U(1)$ gauge invariance to hold in the full modified action, we are led to add the constraint (2.3).

A heuristic argument why this equivalence should hold is that both models, (1.5) and (2.1), have the same number $n_{p h}=\mathbf{8}$ of the physical degrees of freedom. The rigorous proof of the equivalence can be achieved by reducing both systems to the physical degrees of freedom. Namely, choosing light-cone gauge and following the gauge-fixing procedure as in [23], [25], we can show that the actions of the systems (2.1) and (1.5) written in terms of physical variables coincide with each other in the sector with the definite sign of energy. An exposition of this procedure is given in Appendix B.

As for the world-line particle model (1.3) of the HS unfolded formulation, it has also eight physical degrees of freedom and so is expected to be equivalent to the master HS particle model too. Indeed, this model also follows from the master model (2.1) with a particular gauge choice. The spinor constraints (2.5) and the gauge-fixing condition $\zeta^{\alpha} \approx 0$ together with its complex conjugate can be used to eliminate the variables $\zeta^{\alpha}, \pi_{\alpha}$ and their complex conjugates. Then the constraint (2.3) can be used to gauge away $\eta$. The phase and the square of modulus of $\eta$ play the role of the real coordinate and real momentum, respectively

$$
\varphi \equiv-i \ln (\eta / \bar{\eta}), \quad \rho \equiv \eta \bar{\eta}, \quad[\varphi, \rho]_{D}=1
$$

The constraint (2.3) is linear in $\rho$ and generates arbitrary local transformations of $\varphi$. This constraint together with the gauge fixing condition

$$
\chi \equiv \varphi-\text { const } \approx 0
$$

can be used to eliminate the variables $\rho, \varphi$ at expense of the remaining variables $\lambda_{\alpha}, y^{\alpha}, \bar{\lambda}_{\dot{\alpha}}$, $\bar{y}^{\dot{\alpha}}$. Since the condition (2.11) includes only $\varphi$, the brackets for the remaining variables do not change. As a result, we arrive at the system (1.3). 


\subsection{Twistorial formulation of master HS particle}

Before passing to quantization, we present the twistorial formulation of the master HS particle. This formulation can be used to analyze symmetries of the master HS particle model [26]. The symmetry transformations, both finite- and infinite-dimensional, prove to have a transparent form in terms of twistors.

The particle model (2.1) in the twistorial formulation is described by two Weyl spinors $\lambda_{\alpha}$ and $\bar{\mu}^{\dot{\alpha}}$ which form a twistor and by a complex scalar $\xi$. The additional spinor and scalar are introduced by the following twistor transform

$$
\begin{gathered}
\mu^{\alpha}=y^{\alpha}+\bar{\lambda}_{\dot{\beta}}\left(x^{\dot{\beta} \alpha}-i \bar{\zeta}^{\dot{\beta}} \zeta^{\alpha}\right)-2 i \bar{\eta} \zeta^{\alpha}, \quad \bar{\mu}^{\dot{\alpha}}=\bar{y}^{\dot{\alpha}}+\left(x^{\dot{\alpha} \beta}+i \bar{\zeta}^{\dot{\alpha}} \zeta^{\beta}\right) \lambda_{\beta}+2 i \eta \bar{\zeta}^{\dot{\alpha}}, \\
\xi=\eta+\zeta^{\beta} \lambda_{\beta}, \quad \bar{\xi}=\bar{\eta}+\bar{\lambda}_{\dot{\beta}} \bar{\zeta}^{\dot{\beta}} .
\end{gathered}
$$

Using (2.7)-(2.9), one can check that the newly introduced variables are canonical ones

$$
\left[\mu^{\alpha}, \lambda_{\beta}\right]_{D}=\delta_{\beta}^{\alpha}, \quad\left[\bar{\mu}^{\dot{\alpha}}, \bar{\lambda}_{\dot{\beta}}\right]_{D}=\delta_{\dot{\beta}}^{\dot{\alpha}}, \quad[\xi, \bar{\xi}]_{D}=\frac{i}{2} .
$$

Up to the boundary terms, the action (2.1) takes the following form in the twistorial variables

$$
S^{H S-t w}=\int d \tau\left[\lambda_{\alpha} \dot{\mu}^{\alpha}+\bar{\lambda}_{\dot{\alpha}} \dot{\bar{\mu}}^{\dot{\alpha}}+i(\xi \dot{\bar{\xi}}-\dot{\xi} \bar{\xi})-l(U-c)\right] .
$$

Here, the $U(1)$ constraint

$$
U-c \equiv i\left(\mu^{\alpha} \lambda_{\alpha}-\bar{\lambda}_{\dot{\alpha}} \bar{\mu}^{\dot{\alpha}}\right)-2 \xi \bar{\xi}-c \approx 0
$$

is the condition (2.3) rewritten in the twistorial variables (2.12), (2.13).

The twistorial formulation (2.15) of the HS particle reproduces the twistorial HS particle which was considered in $[10,11]$. Due to the constraint $(2.16)$, one can gauge away the variables $\xi, \bar{\xi}$. As in the previous Section, we fix the phase of $\xi$ and eliminate the modulus of $\xi$ by the constraint (2.16). The canonical brackets for the remaining variables $\lambda_{\alpha}, \mu^{\alpha}, \bar{\lambda}_{\dot{\alpha}}, \bar{\mu}^{\dot{\alpha}}$ do not change. As a result, we obtain the system described by the action of ref. $[10,11]$

$$
S_{1}^{H S-t w .}=\int d \tau\left(\lambda_{\alpha} \dot{\mu}^{\alpha}+\bar{\lambda}_{\dot{\alpha}} \dot{\bar{\mu}}^{\dot{\alpha}}\right) .
$$

The twistor system (2.17) can be obtained also directly from the system (1.3) via the following standard twistor transform

$$
\mu^{\alpha}=y^{\alpha}+\bar{\lambda}_{\dot{\beta}} x^{\dot{\beta} \alpha}, \quad \bar{\mu}^{\dot{\alpha}}=\bar{y}^{\dot{\alpha}}+x^{\dot{\alpha} \beta} \lambda_{\beta} .
$$

This provides one more proof of the equivalence of the systems (1.3) and (2.1).

Note that in both twistor transforms, i.e. (2.18) and (2.12), (2.13), the twistor variables are defined in terms of the variables of the "mixed" systems (1.3) and (2.1) which involve both the space-time $(x, \zeta)$ and "twistor-like" $(\lambda, y, \eta)$ variables. This suggests a way of computing some quantities (for example, symmetry generators) in the "mixed" picture, if we know them in the pure twistorial formulation.

For definiteness, we can choose $\operatorname{Arg} \xi=2 k \pi, k \in \mathbf{Z}$ in the gauge fixing condition for the constraint (2.16). Then $\xi$ is real and it is expressed as

$$
\xi=\bar{\xi}=a(\lambda, \bar{\lambda}, \mu, \bar{\mu}), \quad a(\lambda, \bar{\lambda}, \mu, \bar{\mu}) \equiv \sqrt{\frac{i}{2}\left(\mu^{\alpha} \lambda_{\alpha}-\bar{\lambda}_{\dot{\alpha}} \bar{\mu}^{\dot{\alpha}}\right)}
$$

due to $(2.16)$. 


\section{$3 \quad$ First-quantized theory}

\subsection{Canonical quantization of master system}

As shown in Sect. 2.1, after resolving the second class constraints (2.6) through the Dirac procedure, in the system (2.1) there remain only first class constraints. Therefore the quantization procedure is straightforward in this approach ${ }^{4}$.

We use the coordinate representation of the canonical brackets $(2.7)-(2.9)$ in which the momentum operators have the following realization

$$
\begin{gathered}
\hat{P}_{\alpha \dot{\alpha}}=-i \partial / \partial x^{\dot{\alpha} \alpha} \equiv-i \partial_{\alpha \dot{\alpha}}, \quad \hat{\pi}_{\alpha}=-i \partial / \partial \zeta^{\alpha} \equiv-i \partial_{\alpha}, \quad \hat{\bar{\pi}}_{\dot{\alpha}}=-i \partial / \partial \bar{\zeta}^{\dot{\alpha}} \equiv-i \bar{\partial}_{\dot{\alpha}}, \\
\hat{\lambda}_{\alpha}=-i \partial / \partial y^{\alpha}, \quad \hat{\bar{\lambda}}_{\dot{\alpha}}=-i \partial / \partial \bar{y}^{\dot{\alpha}}, \quad \hat{\bar{\eta}}=\frac{1}{2} \partial / \partial \eta .
\end{gathered}
$$

The constraints $(2.3)-(2.5)$ become the set of equations for the wave function $F^{(q)}(x, \zeta, \bar{\zeta}, y, \bar{y}, \eta)$

$$
\begin{gathered}
\left(\partial_{\alpha \dot{\beta}}+i \frac{\partial}{\partial y^{\alpha}} \frac{\partial}{\partial \bar{y}^{\dot{\beta}}}\right) F^{(q)}=0 \\
\left(D_{\alpha}+\frac{\partial}{\partial \eta} \frac{\partial}{\partial y^{\alpha}}\right) F^{(q)}=0 \\
\left(\bar{D}_{\dot{\alpha}}-2 \eta \frac{\partial}{\partial \bar{y}^{\dot{\alpha}}}\right) F^{(q)}=0 \\
\left(y^{\alpha} \frac{\partial}{\partial y^{\alpha}}-\bar{y}^{\dot{\alpha}} \frac{\partial}{\partial \bar{y}^{\dot{\alpha}}}-\eta \frac{\partial}{\partial \eta}\right) F^{(q)}=q F^{(q)} .
\end{gathered}
$$

Here, the operators

$$
D_{\alpha}=-i\left(\partial_{\alpha}+i \partial_{\alpha \dot{\alpha}} \bar{\zeta}^{\dot{\alpha}}\right), \quad \bar{D}_{\dot{\alpha}}=-i\left(\bar{\partial}_{\dot{\alpha}}-i \zeta^{\alpha} \partial_{\alpha \dot{\alpha}}\right)
$$

are quantum counterparts of the "covariant momenta" (1.7).

The external $U(1)$ charge $q$ defined by (3.6) is the quantum counterpart of the "classical" constant $c$ present in the constraint (2.3), now with the ordering ambiguities taken into account. Eq. (3.6) implies $U(1)$ covariance of the wave function

$$
F^{(q)}\left(x, \zeta, \bar{\zeta}, e^{i \varphi} y, e^{-i \varphi} \bar{y}, e^{-i \varphi} \eta\right)=e^{q i \varphi} F^{(q)}(x, \zeta, \bar{\zeta}, y, \bar{y}, \eta)
$$

Requiring $F^{(q)}$ to be single-valued restricts $q$ to the integer values. As follows from (3.7), the $U(1)$ charges of the coordinates $y_{\alpha}$ and $\bar{y}_{\dot{\alpha}}$ are opposite while $\eta$ has the same charge as $\bar{y}_{\dot{\alpha}}$. The rest of coordinates are neutral. It is important to note that this $U(1)$ covariance just implies that any monomial of the charged coordinates in the series expansion of the wave function $F^{(q)}$ has the same fixed charge $q$ and it does not entail any $U(1)$ transformation of the coefficient fields. In this respect $q$ resembles the "harmonic $U(1)$ charge" of the harmonic superspace approach $[27,28]$ and the operator in (3.6) is an analog of the $U(1)$ charge-counting operator

\footnotetext{
${ }^{4}$ In Appendix A we demonstrate that an equivalent quantization can be performed using the Gupta-Bleuler method in which the variables $\eta$ and $\bar{\eta}$ are treated as mutually conjugated in the ordinary sense and only one of the constraints (2.6) is imposed on the complex wave function.
} 
$D^{0}$ in this approach. As we shall see, $\frac{q}{2}$ always coincides with the lowest helicity in the infinite tower of helicities comprised by the wave function $F^{(q)}$.

Before presenting the solution of eqs. (3.3)-(3.6), let us mention one more notable feature of this system. Though it involves the Vasiliev-type unfolded vector equation (3.3), the latter follows from the spinorial eqs. (3.4) and (3.5) as their integrability condition ${ }^{5}$. So the basic independent equations of the system (3.3)-(3.6) are the bosonic spinorial equations (3.4), (3.5) and the $U(1)$ condition (3.6).

Let us now solve eqs. (3.4)-(3.6). One can do this in several equivalent ways.

It is most convenient to work in the variables $\zeta^{\alpha}, \bar{\zeta}^{\dot{\alpha}}, y^{\alpha}, \eta$ and

$$
x_{L}^{\dot{\alpha} \alpha}=x^{\dot{\alpha} \alpha}+i \bar{\zeta}^{\dot{\alpha}} \zeta^{\alpha}, \quad \bar{y}_{L}^{\dot{\alpha}}=\bar{y}^{\dot{\alpha}}+2 i \eta \bar{\zeta}^{\dot{\alpha}} .
$$

In these new variables eqs. (3.4)-(3.6) take the form

$$
\begin{gathered}
{\left[-i\left(\partial_{\alpha}+i \frac{\partial}{\partial \eta} \frac{\partial}{\partial y^{\alpha}}\right)+2 \bar{\zeta}^{\dot{\alpha}}\left(\partial_{L \alpha \dot{\alpha}}+i \frac{\partial}{\partial y^{\alpha}} \frac{\partial}{\partial \bar{y}_{L}^{\dot{\alpha}}}\right)\right] F^{(q)}=0} \\
\bar{\partial}_{\dot{\alpha}} F^{(q)}=0 \\
\left(y^{\alpha} \frac{\partial}{\partial y^{\alpha}}-\bar{y}_{L}^{\dot{\alpha}} \frac{\partial}{\partial \bar{y}_{L}^{\dot{\alpha}}}-\eta \frac{\partial}{\partial \eta}\right) F^{(q)}=q F^{(q)} .
\end{gathered}
$$

Eq. (3.10) is the bosonic chirality condition stating that $F^{(q)}$ does not depend on $\bar{\zeta}^{\dot{\alpha}}$ in the new variables,

$$
F^{(q)}=F^{(q)}\left(x_{L}, \zeta, y, \bar{y}_{L}, \eta\right)
$$

Then eq. (3.9) amounts to two equations

$$
\left(\partial_{\alpha}+i \frac{\partial}{\partial \eta} \frac{\partial}{\partial y^{\alpha}}\right) F^{(q)}=0
$$

and

$$
\left(\partial_{L \alpha \dot{\alpha}}+i \frac{\partial}{\partial y^{\alpha}} \frac{\partial}{\partial \bar{y}_{L}^{\dot{\alpha}}}\right) F^{(q)}=0 .
$$

The important corollaries of eqs. (3.13) and (3.14) are the following equations

$$
\partial_{L}^{\dot{\alpha} \alpha} \partial_{\alpha} F^{(q)}=0, \quad \partial_{L}^{\dot{\alpha} \alpha} \partial_{L \alpha \dot{\alpha}} F^{(q)}=0
$$

where we exploited the property $\frac{\partial}{\partial y^{\alpha}} \frac{\partial}{\partial y_{\alpha}} \equiv 0$.

As the next step, we extract some further consequences of eq. (3.13). By analogy with (1.9) we assume the polynomial dependence of the field (3.12) on $\zeta^{\alpha}$

$$
F^{(q)}\left(x_{L}, \zeta, y, \bar{y}_{L}, \eta\right)=\sum_{n=0}^{\infty} \zeta^{\alpha_{1}} \ldots \zeta^{\alpha_{n}} \Phi_{\alpha_{1} \ldots \alpha_{n}}^{(q)}\left(x_{L}, y, \bar{y}_{L}, \eta\right)
$$

Eq. (3.13) expresses all the coefficients in this expansion as derivatives of the first coefficient $\Phi^{(q)}\left(x_{L}, y, \bar{y}_{L}, \eta\right)$

$$
\Phi_{\alpha_{1} \ldots \alpha_{n}}^{(q)}=\frac{(-i)^{n}}{n !} \frac{\partial^{n}}{\partial \eta^{n}} \frac{\partial}{\partial y^{\alpha_{1}}} \cdots \frac{\partial}{\partial y^{\alpha_{n}}} \Phi^{(q)}\left(x_{L}, y, \bar{y}_{L}, \eta\right)
$$

\footnotetext{
${ }^{5}$ One can say that (3.4) and (3.5) play the role of bosonic "square root" of (3.3).
} 
Thus we have reduced the initial system of equations to the two equations on the function $\Phi^{(q)}$
(a) $\left(\partial_{L \alpha \dot{\alpha}}+i \frac{\partial}{\partial y^{\alpha}} \frac{\partial}{\partial \bar{y}_{L}^{\dot{\alpha}}}\right) \Phi^{(q)}=0$
(b) $\left(y^{\alpha} \frac{\partial}{\partial y^{\alpha}}-\bar{y}_{L}^{\dot{\alpha}} \frac{\partial}{\partial \bar{y}_{L}^{\dot{\alpha}}}-\eta \frac{\partial}{\partial \eta}\right) \Phi^{(q)}=q \Phi^{(q)}$.

Like in the previous cases, we assume that $\Phi^{(q)}\left(x_{L}, y, \bar{y}_{L}, \eta\right)$ has a non-singular polynomial expansion over the additional coordinates. Then eq. (3.18b) implies

$$
\Phi^{(q)}\left(x_{L}, y, \bar{y}_{L}, \eta\right)=\sum_{k=0}^{\infty} \eta^{k} \varphi^{(q+k)}\left(x_{L}, y, \bar{y}_{L}\right)
$$

and

$$
\left(y^{\alpha} \frac{\partial}{\partial y^{\alpha}}-\bar{y}_{L}^{\dot{\alpha}} \frac{\partial}{\partial \bar{y}_{L}^{\dot{\alpha}}}\right) \varphi^{(q+k)}=(q+k) \varphi^{(q+k)}
$$

(recall that $\eta$ carries the negative unit $U(1)$ charge). The reduced $U(1)$ condition (3.20) fixes the $y, \bar{y}$ dependence of the functions $\varphi^{(q+k)}$ as

$$
\varphi^{(q+k)}\left(x_{L}, y, \bar{y}_{L}\right)= \begin{cases}\sum_{n=0}^{\infty} y^{\alpha_{1}} \ldots y^{\alpha_{q+k+n}} \bar{y}_{L}^{\dot{\beta}_{1}} \ldots \bar{y}_{L}^{\dot{\beta}_{n}} \phi_{\alpha_{1} \ldots \alpha_{q+k+n} \dot{\beta}_{1} \ldots \dot{\beta}_{n}}\left(x_{L}\right), & (q+k) \geq 0, \\ \sum_{n=0}^{\infty} y^{\alpha_{1}} \ldots y^{\alpha_{n}} \bar{y}_{L}^{\dot{\beta}_{1}} \ldots \bar{y}_{L}^{\dot{\beta}_{|q+k|+n}} \phi_{\alpha_{1} \ldots \alpha_{n} \dot{\beta}_{1} \ldots \dot{\beta}_{|q+k|+n}}\left(x_{L}\right), & (q+k)<0 .\end{cases}
$$

It remains to reveal the restrictions imposed on the fields $\varphi^{(q+k)}$ by the remaining unfolded equation (3.18a). We shall separately consider the cases $q=0, q>0$ and $q<0$.

\subsection{1 $\mathrm{q}=0$}

It is easy to see that in this case eq. (3.18a) expresses all the fields $\phi_{\alpha_{1} \ldots \alpha_{k+n} \dot{\beta}_{1} \ldots \dot{\beta}_{n}}$ with $n>0$ in $\varphi^{(k)}$ as $x$-derivatives of the lowest component, the self-dual field $\phi_{\alpha_{1} \ldots \alpha_{k}}$. The latter field satisfies Dirac and Klein-Gordon equations

$$
\partial^{\dot{\beta} \alpha_{1}} \phi_{\alpha_{1} \ldots \alpha_{k}}=0, \quad \square \phi=0
$$

also in consequence of the same eq. (3.18a).

Thus the space of physical states of the model is spanned by the complex self-dual field strengths $\phi_{\alpha_{1} \ldots \alpha_{k}}, k=0,1,2, \ldots$, of the massless particles of all integer and half-integer helicities.

It is to the point here to make comparison with the Vasiliev approach $[5,16]$. The field $\Phi^{(0)}$ and its conjugate $\bar{\Phi}^{(0)}\left(x_{R}, y_{R}, \bar{y}, \bar{\eta}\right)$ (with $x_{R}=\overline{\left(x_{L}\right)}, y_{R}=\overline{\left(\bar{y}_{L}\right)}$ ) encompass the same set of fields as the real field (1.2), the only difference being the presence of complex scalar field in our case compared to a real such field in (1.2). Indeed, the conjugated HS field $\bar{\Phi}^{(0)}\left(x_{R}, y_{R}, \bar{y}, \bar{\eta}\right)$ describes anti-self-dual fields which are complex conjugates of the self-dual fields $\phi_{\alpha_{1} \ldots \alpha_{k}}$. So no any doubling of higher spins occurs in our case, like in the original unfolded formulation. Formally, the HS field (1.2) can be recovered as the sum $\Phi=\Phi^{(0)}+\bar{\Phi}^{(0)}$ at the "point" $\eta=1$.

Thus we conclude that the higher spin multiplet comprised by the field (1.2) is a particular case of the multiplets arising after quantization of the master HS particle, corresponding to the choice $q=0$ for the external $U(1)$ charge. 


\subsection{2 $\mathrm{q}>0$}

Like in the $q=0$ case, for the generic positive $q$ eq. (3.18a) expresses the fields $\phi_{\alpha_{1} \ldots \alpha_{q+k+n} \dot{\beta}_{1} \ldots \dot{\beta}_{n}}$ with $n>0$ in (3.21) in terms of the $\partial_{\alpha \dot{\alpha}}$-derivatives of the self-dual fields $\phi_{\alpha_{1} \ldots \alpha_{q+k}}$. Also, the same eq. (3.14) yields Dirac equations for the independent fields

$$
\partial^{\dot{\beta} \alpha_{1}} \phi_{\alpha_{1} \ldots \alpha_{q+k}}=0, \quad k=0,1,2, \ldots
$$

Thus the space of physical states of the model is spanned by the self-dual field strengths of the massless particles with helicities $\frac{q}{2}, \frac{q}{2}+\frac{1}{2}, \frac{q}{2}+1, \ldots$ We observe that the scalar field is absent in the spectrum for non-zero positive $q$. Once again, the relevant HS multiplet is fully characterized by the value of $q$.

\subsection{3 $\mathrm{q}<0$}

For the negative values of $q$ the expansion (3.19) can be conveniently rewritten as

$$
\begin{aligned}
\Phi^{(q)}\left(x_{L}, y, \bar{y}_{L}, \eta\right)= & \eta^{|q|} \tilde{\Phi}^{(0)}\left(x_{L}, y, \bar{y}_{L}, \eta\right)+ \\
& +\sum_{k=1}^{|q|} \sum_{m=0}^{\infty} \eta^{|q|-k} y^{\alpha_{1}} \ldots y^{\alpha_{m}} \bar{y}_{L}^{\dot{\beta}_{1}} \ldots \bar{y}_{L}^{\dot{\beta}_{m+k}} \phi_{\alpha_{1} \ldots \alpha_{m} \dot{\beta}_{1} \ldots \dot{\beta}_{m+k}}\left(x_{L}\right) .
\end{aligned}
$$

Thus in this case we deal with the HS field $\tilde{\Phi}^{(0)}$ having the same helicity contents as the $q=0$ multiplet and an additional term involving the space-time fields $\phi_{\alpha_{1} \ldots \alpha_{m} \dot{\beta}_{1} \ldots \dot{\beta}_{m+k}}$. The set of independent fields in this expansion consists of self-dual fields $\phi_{\alpha_{1} \ldots \alpha_{k}}, k=0,1,2, \ldots$ present in the HS field $\Phi^{(0)}$ and anti-self-dual fields $\phi_{\dot{\beta}_{1} \ldots \dot{\beta}_{k}}, k=1, \ldots,|q|$. Eq. (3.18a) expresses all other space-time fields as space-time derivatives of these basic ones. Eq. (3.18a) also implies the Dirac and Klein-Gordon equations for the basic fields.

Thus, for $q<0$ physical fields in the spectrum describe massless particles with all positive helicities starting from the zero one, and also a finite number of massless states with negative helicities $-\frac{1}{2},-1, \ldots,-\frac{|q|}{2}$. This HS multiplet could be naturally called "helicity-flip" multiplet. Note that, being considered together with its conjugate, this multiplet reveals a partial doubling of fields with a given helicity, the phenomenon which is absent in the previous two cases.

We would like to point out that the multiplets listed above were derived under the assumption that the $\eta$-expansions of the wave functions are regular at the origin and so are given by power series over $\eta$. Alternatively, we could consider the wave functions which are regular at $\infty$ and so are represented by series over $1 / \eta$. In this case we would obtain basically the same set of states (up to the interchange of positive and negative helicities). If one gives up the regularity assumption, it is impossible to avoid the doubling of helicities for any value of $q$.

\subsection{Alternative description}

Here we establish the link with the HS description suggested by the quantization of the particle with bosonic "supersymmetry" [17].

We start with the case $q=0$ and consider at first the expansion of the field $F^{(q)}$ defined in $(3.12)$ with respect to the coordinates $y, \bar{y}_{L}$ and $\eta$

$$
F^{(0)}\left(x_{L}, \zeta, y, \bar{y}_{L}, \eta\right)=\sum_{k=0}^{\infty} \sum_{n=0}^{\infty} \eta^{k} y^{\alpha_{1}} \ldots y^{\alpha_{k+n}} \bar{y}_{L}^{\dot{\alpha}_{1}} \ldots \bar{y}_{L}^{\dot{\alpha}_{n}} \Psi_{\alpha_{1} \ldots \alpha_{k+n} \dot{\alpha}_{1} \ldots \dot{\alpha}_{n}}\left(x_{L}, \zeta\right)
$$


where we have already taken care of eq. (3.11). It remains to take into account eqs. (3.13) and (3.14). They express all the coefficient fields in (3.25) as derivatives of the lowest coefficient $\Psi\left(x_{L}, \zeta\right)$ :

$$
\Psi_{\alpha_{1} \ldots \alpha_{k} \alpha_{k+1} \ldots \alpha_{k+n} \dot{\alpha}_{1} \ldots \dot{\alpha}_{n}}=\frac{i^{k+n}}{k !(n+k) ! n !} \partial_{\alpha_{1}} \ldots \partial_{\alpha_{k}} \partial_{L \alpha_{k+1} \dot{\alpha}_{1}} \ldots \partial_{L \alpha_{k+n} \dot{\alpha}_{n}} \Psi
$$

The field $\Psi\left(x_{L}, \zeta\right)$ satisfies eqs. (3.15) and it is exactly the chiral HS field (1.9) of the paper [17]. It comprises the same irreducible HS $q=0$ multiplet as $\Phi^{(0)}\left(x_{L}, y, \bar{y}_{L}, \eta\right)$. The possibility to describe the same multiplet in two equivalent ways is just the quantum version of equivalence between the HS particles (1.3) and (1.5) which both follow from the master HS particle. In a sense, the description by the field $\Psi\left(x_{L}, \zeta\right)$ is more economical since this quantity collects, in its $\zeta^{\alpha}$ expansion, just the independent space-time self-dual fields (anti-self-dual fields are contained in the complex-conjugated function $\left.\bar{\Psi}\left(x_{R}, \bar{\zeta}\right)\right)$.

Let us also consider the alternative description of the $q>0$ HS multiplets. The corresponding counterpart of the $q=0$ expansion is

$$
F^{(q)}\left(x_{L}, \zeta, y, \bar{y}_{L}, \eta\right)=\sum_{k=0}^{\infty} \sum_{n=0}^{\infty} \eta^{k} y^{\alpha_{1}} \ldots y^{\alpha_{q+k+n}} \bar{y}_{L}^{\dot{\beta}_{1}} \ldots \bar{y}_{L}^{\dot{\beta}_{n}} \Psi_{\alpha_{1} \ldots \alpha_{q+k+n} \dot{\beta}_{1} \ldots \dot{\beta}_{n}}\left(x_{L}, \zeta\right)
$$

where we have already taken account of the $U(1)$ condition (3.11). Then eqs. (3.13) and (3.14) lead to the following expressions for the coefficients in $(3.27)$

$$
\Psi_{\alpha_{1} \ldots \alpha_{q} \alpha_{q+1} \ldots \alpha_{q+k+n} \dot{\beta}_{1} \ldots \dot{\beta}_{n}}=\frac{i^{k+n} q !}{k ! n !(q+n+k) !} \partial_{\alpha_{q+1}} \ldots \partial_{\alpha_{q+k}} \partial_{L \alpha_{q+k+1} \dot{\beta}_{1}} \ldots \partial_{L \alpha_{q+k+n} \dot{\beta}_{n}} \Psi_{\alpha_{1} \ldots \alpha_{q}}
$$

Thus all component fields are expressed in terms of the even counterpart of $N=1$ chiral field with external indices $\Psi_{\alpha_{1} \ldots \alpha_{q}}\left(x_{L}, \zeta\right)$.

Also as a consequence of eqs. (3.13) and (3.14), this field proves to be subjected to eqs. (3.15) and also to the equations

$$
\partial^{\alpha_{1}} \Psi_{\alpha_{1} \ldots \alpha_{q}}=0, \quad \partial_{L}^{\dot{\alpha} \alpha_{1}} \Psi_{\alpha_{1} \ldots \alpha_{q}}=0
$$

Expanding the field $\Psi_{\alpha_{1} \ldots \alpha_{q}}$ in powers of $\zeta_{\alpha}$

$$
\Psi_{\alpha_{1} \ldots \alpha_{q}}\left(x_{L}, \zeta\right)=\sum_{n=0}^{\infty} \zeta^{\beta_{1}} \ldots \zeta^{\beta_{n}} \psi_{\alpha_{1} \ldots \alpha_{q} \beta_{1} \ldots \beta_{n}}\left(x_{L}\right),
$$

we observe that all component fields in this expansion are totally symmetric in the spinor indices due to the first equation in (3.29). As a consequence of eqs. (3.15) and the second equation in (3.29), all component fields satisfy Dirac equation. Therefore, the HS field (3.30) describes the same physical spectrum as in eq. (3.23). Note that the bosonic chiral "superfields" with extra indices were not considered in [17]. It is the master HS particle framework which suggests the importance of considering such superfields as providing an alternative description of the $q>0$ HS multiplets.

For the $q<0$ case, a formulation in terms of the fields depending on $x_{L}$ and $\zeta$ can be also easily constructed. It operates with the HS field (1.9) comprising the infinite set of positive helicities and few extra anti-self-dual fields $\phi_{\dot{\beta}_{1} \ldots \dot{\beta}_{k}}\left(x_{L}\right)(k=1, \ldots,|q|)$. 


\subsection{Quantization in twistor formulation: twistor transform for $\mathrm{HS}$ fields}

In the remainder of this Section we show that quantization of the considered model in the twistorial formulation (2.15) gives rise to the same results.

In the "twistorial representation" ${ }^{6}$, when $\lambda_{\alpha}, \bar{\mu}^{\dot{\alpha}}$ and $\xi$ are diagonal and

$$
\hat{\mu}^{\alpha}=i \partial / \partial \lambda_{\alpha}, \quad \hat{\bar{\lambda}}_{\dot{\alpha}}=-i \partial / \partial \bar{\mu}^{\dot{\alpha}}, \quad \hat{\bar{\xi}}=\frac{1}{2} \partial / \partial \xi,
$$

the twistorial wave function

$$
G^{(q-2)}(\lambda, \bar{\mu}, \xi)
$$

satisfies the quantum counterpart of the constraints (2.16)

$$
\left(-\lambda_{\alpha} \frac{\partial}{\partial \lambda_{\alpha}}-\bar{\mu}^{\dot{\alpha}} \frac{\partial}{\partial \bar{\mu}^{\dot{\alpha}}}-\xi \frac{\partial}{\partial \xi}\right) G^{(q-2)}=(q-2) G^{(q-2)} .
$$

The wave function in the space-time description is derived by analogy with the standard twistor approach [29]. One substitutes the incidence conditions (2.12) and (2.13) for the variables $\bar{\mu}^{\dot{\alpha}}$ and $\xi$ in the wave function (3.31) and performs a Fourier-type integral transformation from $\lambda_{\alpha}$ to its canonically conjugated variable $y_{\alpha}$

$$
F^{(q)}\left(x_{L}, \zeta, y, \bar{y}_{L}, \eta\right)=\int d^{2} \lambda e^{i y^{\alpha} \lambda_{\alpha}} G^{(q-2)}\left(\lambda_{\alpha}, \bar{y}_{L}^{\dot{\alpha}}+x_{L}^{\dot{\alpha} \beta} \lambda_{\beta}, \eta+\zeta^{\beta} \lambda_{\beta}\right) .
$$

Note that the variables $x_{L}$ and $\bar{y}_{L}$ defined in (3.8) already appeared in the twistor transform (2.12) for $\bar{\mu}$. The integrand in (3.33) includes the Fourier exponent in contrast to the Penrose integral transform [29].

Using the particular dependence of the twistorial field $G^{(q-2)}$ on the involved co-ordinates, it is easy to check that the field $F^{(q)}$ defined by (3.33) automatically satisfies eqs. (3.13) and (3.14). Also, homogeneity condition (3.11) follows from eq. (3.32). Thus, the twistorial formulation solves eqs. (3.11), (3.13) and (3.14) for the field (3.12) in terms of the unconstrained "prepotential" field (3.31).

\section{Summary and outlook}

In this paper we have constructed a "master" model of HS particle. It possesses several notable features which are summarized below together with some of their corollaries:

- Both the unfolded HS particle and the HS particle with the even "supersymmetry" can be reproduced from the master model upon some gauge fixings.

- Hamiltonian formulation of the master system involves only first class constraints that, in particular, makes it possible to directly pass to the first-quantized model.

- The twistor formulation of the master system is a HS generalization of the well-known twistor formulation of massless particles with fixed helicities.

\footnotetext{
${ }^{6}$ The spinors $\lambda_{\alpha}$ and $\bar{\mu}^{\dot{\alpha}}$ together form a unit twistor; they both are diagonal in the "twistorial representation" [29].
} 
- After quantization, the set of equations for the HS wave function in an extended space includes the Vasiliev-type unfolded equation. But the latter turns out to be a consequence of some basic spinorial equations related to the even "supersymmetry" of the model.

- The HS fields respect a local $U(1)$ symmetry which is similar to the $U(1)$ covariance of the harmonic approach [27, 28]. Crucial for maintaining this covariance is the holomorphic dependence of the HS wave function on the new complex bosonic coordinate $\eta$.

- Depending on their external $U(1)$ charge $q$, the HS fields in the extended space accommodate different HS multiplets of ordinary $4 D$ fields. The all-helicity HS multiplet of the unfolded formulation (with a complex scalar field) is recovered as the $q=0$ multiplet and its conjugate, and also some new HS multiplets with $q \neq 0$ emerge. For $q>0$ they are spanned by the self-dual field strengths of growing positive helicities, starting from $\frac{q}{2}$. The $q<0$ multiplets show up an interesting "spin-flip" feature: they include self-dual fields of all positive helicities, as well as a finite number of anti-self-dual fields with negative helicities. The complementary helicities are accommodated by complex conjugated wave functions.

- The unfolded formulation of HS fields and their formulation with explicit bosonic "supersymmetry" are equivalent to each other as they correspond to different ways of solving the same master system of HS equations. For the new HS multiplets (with $q \neq 0$ ) this equivalence implies, besides the appropriate unfolded formulation, also the description with the explicit bosonic "supersymmetry".

- The master HS equations in the twistorial formulation can be solved in terms of unconstrained twistor prepotential field.

In the rest of this Section we announce some results of studying symmetries of the master HS particle model and the new HS equations associated with it. More details will be given in [26].

Since the master model encompasses both the unfolded HS particle (1.3) and the HS particle with explicit even "supersymmetry" (1.5), it should respect the symmetries inherent to both these systems. Moreover, the equivalence of these models implies that they possess the same full sets of symmetries, though realized in different ways. Some symmetries can be explicit in the first model, but hidden in the second one, and vice versa.

Let us illustrate this on the example of the invariance of the system (1.5) under the even "supersymmetry" translations [17]-[19]

$$
\delta x^{\dot{\alpha} \alpha}=i\left(\bar{\epsilon}^{\dot{\alpha}} \zeta^{\alpha}-\bar{\zeta}^{\dot{\alpha}} \epsilon^{\alpha}\right), \quad \delta \zeta^{\alpha}=\epsilon^{\alpha}, \quad \delta \bar{\zeta}^{\dot{\alpha}}=\bar{\epsilon}^{\dot{\alpha}},
$$

where $\epsilon^{\alpha}$ is a commuting Weyl spinor. Master system (2.1) is invariant under the transformations (4.1) too. The variables $\lambda_{\alpha}, y^{\alpha}, \eta$ and their complex conjugates are inert under the even "supersymmetry" translations.

The symmetry (4.1) is hidden in the particle model (1.3). However, it becomes manifest in the twistorial formulations $(2.15),(2.17)^{7}$. In the twistorial formulation (2.15) of the master system, the transformations (4.1) act as

$$
\delta \lambda_{\alpha}=\epsilon^{\alpha} \lambda_{\alpha}, \quad \delta \mu^{\alpha}=-2 i \bar{\xi} \epsilon^{\alpha} \quad \text { and c. c. . }
$$

\footnotetext{
${ }^{7}$ The advantage of using twistors $[29,22]$ for the analysis of symmetries of relativistic particles was demonstrated in [30] (for recent developments in the twistor approach see [31] and references therein).
} 
They are generated by the following charges

$$
R_{\alpha}=-2 i \bar{\xi} \lambda_{\alpha}, \quad \bar{R}_{\dot{\alpha}}=2 i \xi \bar{\lambda}_{\dot{\alpha}} .
$$

The twistorial model (2.17) is obtained from the model (2.15) via the gauge fixing. Therefore, bosonic "supersymmetry" translations in the model (2.17) should be accompanied by the appropriate compensating gauge transformation in order to preserve the gauge-fixing condition. As a result we obtain the following (nonlinear) even "supersymmetry" transformations of the variables in the model $(2.17)$

$$
\delta \lambda_{\alpha}=-\frac{1}{2} a^{-1}(\epsilon \lambda-\bar{\lambda} \bar{\epsilon}) \lambda_{\alpha}, \quad \delta \mu^{\alpha}=\frac{1}{2} a^{-1}(\epsilon \lambda-\bar{\lambda} \bar{\epsilon}) \mu^{\alpha}-2 i a \epsilon^{\alpha},
$$

where $a(\lambda, \bar{\lambda}, \mu, \bar{\mu})$ was defined in (2.19). It is straightforward to check that these transformations leave invariant the action (2.17). Correspondingly, the bosonic "supersymmetry" generators in the model (2.17) are

$$
R_{\alpha}=-2 i a \lambda_{\alpha}, \quad \bar{R}_{\dot{\alpha}}=2 i a \bar{\lambda}_{\dot{\alpha}} .
$$

The model (2.17) has also $S p(8)$ symmetry. It is generated by the full set of bilinear products of the spinors $\lambda_{\alpha}, \bar{\lambda}_{\dot{\alpha}}, \mu^{\alpha}$ and $\bar{\mu}^{\dot{\alpha}}$. The closure of $S p(8)$ with bosonic "supersymmetry" generated by (4.5), as well as with an infinite sequence of phase transformations generated by arbitrary powers of $a(\lambda, \bar{\lambda}, \mu, \bar{\mu})$, constitutes the full infinite-dimensional symmetry of the model (2.17). This infinite-dimensional invariance can be interpreted as simplectic diffeomorphisms [32]-[35]. A similar interpretation of an infinite-dimensional symmetry in the case of the twistor particle with a fixed helicity was given in [30].

As follows from the form of the generators (4.5), on the HS fields (1.2) depending on the coordinates $x, y, \bar{y}$ the bosonic "supersymmetry" transformations are realized by highly non-linear (and presumably non-local) transformations. Remarkably, on the fields $\Phi^{(q)}$ defined in (3.19) and depending on the extra bosonic co-ordinate $\eta$, the even "supersymmetry" generators have the simple form

$$
R_{\alpha}=-\frac{\partial}{\partial \eta} \frac{\partial}{\partial y^{\alpha}}, \quad \bar{R}_{\dot{\alpha}}=2 \eta \frac{\partial}{\partial \bar{y}^{\dot{\alpha}}} .
$$

They close just on the $x$-translations with the generator $\sim \partial_{\alpha \dot{\alpha}}$, taking into account the unfolded equation (3.18a). This example clearly shows the important role the co-ordinate $\eta$ plays in revealing symmetries of the master system of HS equations. It is worthwhile to notice that the transformations with the generators (4.6) mix up fields with different helicities from different coefficients $\varphi^{(q+k)}$ in (3.19).

As for further directions of the study, constructing supersymmetric extensions of the proposed model (with standard "odd" supersymmetries) is one of the urgent problems. Supersymmetric $N=1$ HS theories constructed on the basis of theories with bosonic "supersymmetry" are expected to be the simplest HS theories respecting the fundamental notion of chirality which underlies the geometric approach to $N=1$ supergravity [36]. The existence of a chiral limit seems to be crucial for any satisfactory HS superfield theory including HS generalizations of $N=1$ supergravity [14,37].

Another issue to be further explored is related to the presence of commuting spinorial variables in most of versions of the HS field theory. In our opinion, the question of how to ensure the correct statistics of the component fields in the expansions of HS field over even 
spinors (see e.g. the power expansion (1.2) and other analogical expansions) is still open (see e.g. some comments in [17]). We expect that the consideration of the HS models having both even and odd supersymmetries could provide a clue to resolving this problem.

Finally, it would be interesting to investigate possible implications of the additional scalar coordinate $\eta$ (which plays a crucial role in the master system) in the geometry of interacting HS fields and in the problem of constructing the relevant actions. Also, it is desirable to see how the master system could be extended to incorporate the tensorial coordinates.

\section{Acknowledgments}

We thank J.A. de Azcárraga, I. Bandos, J. Lukierski, D. Sorokin and M. Vasiliev for interest in the work and useful remarks. E.I. thanks the Physical Department of the National Technical University in Athens for the kind hospitality at the final stage of this work. A partial support from the RFBR grant 06-02-16684 and a grant of Heisenberg-Landau program is acknowledged.

\section{Appendix A: Analysis of the constraints and Gupta- Bleuler quantization}

In this Appendix we carry out more detailed Hamiltonian analysis of the master system (2.1) and show that it can be equivalently quantized by the Gupta-Bleuler (or "analytic") method [21].

Like in any system with one time derivative on fields, the expressions for all momenta give the constraints. Namely, we obtain the primary constraints (2.4), (2.5) and the constraints

$$
\begin{gathered}
g \equiv p_{\eta}+i \bar{\eta} \approx 0, \quad \bar{g} \equiv \bar{p}_{\eta}-i \eta \approx 0, \\
p_{l} \approx 0
\end{gathered}
$$

where $p_{\eta}, \bar{p}_{\eta}$ and $p_{l}$ are the conjugate momenta for $\eta, \bar{\eta}$ and $l$, respectively. We at once treat the variable $\lambda_{\alpha}$ and $\bar{\lambda}_{\dot{\alpha}}$ as momenta for $y^{\alpha}$ and $\bar{y}^{\dot{\alpha}}$. The total Hamiltonian

$$
H_{T}=l(\mathcal{N}-c)+\Lambda^{\alpha \dot{\alpha}} T_{\alpha \dot{\alpha}}+\Lambda^{\alpha} \mathcal{D}_{\alpha}+\overline{\mathcal{D}}_{\dot{\alpha}} \bar{\Lambda}^{\dot{\alpha}}+v g+\bar{v} \bar{g}+\gamma p_{l}
$$

is a sum of the canonical Hamiltonian $H_{0}=l(\mathcal{N}-c)$ and a linear combination of the constraints with Lagrange multipliers $\Lambda^{\alpha \dot{\alpha}}, \ldots, \gamma$.

The requirement of preservation of the constraint (A.2) produces the constraint (2.3) as the secondary constraint. Due to the constraint (A.2) the variable $l$ has the pure gauge character. It plays the role of Lagrange multiplier for the constraint (2.3).

The preservation of the constraints (A.1) gives rise to the elimination of a part of Lagrange multipliers in terms of the remaining ones

$$
v=-\Lambda^{\alpha} \lambda_{\alpha}-i l \eta, \quad \bar{v}=-\bar{\lambda}_{\dot{\alpha}} \bar{\Lambda}^{\dot{\alpha}}+i l \bar{\eta} .
$$

Inserting these expressions in the Hamiltonian (A.3), we obtain

$$
H_{T}=l(\tilde{\mathcal{N}}-c)+\Lambda^{\alpha \dot{\alpha}} T_{\alpha \dot{\alpha}}+\Lambda^{\alpha} \tilde{\mathcal{D}}_{\alpha}+\overline{\tilde{\mathcal{D}}}_{\dot{\alpha}} \bar{\Lambda}^{\dot{\alpha}}
$$


where the new constraints are

$$
\begin{gathered}
\tilde{\mathcal{N}}-c \equiv \mathcal{N}-i\left(\eta g-\bar{\eta} \bar{g}-c=i\left(y^{\alpha} \lambda_{\alpha}-\bar{\lambda}_{\dot{\alpha}} \bar{y}^{\dot{\alpha}}\right)-i\left(\eta p_{\eta}-\bar{\eta} \bar{p}_{\eta}\right)-c \approx 0\right. \\
\tilde{\mathcal{D}}_{\alpha} \equiv \mathcal{D}_{\alpha}-g \lambda_{\alpha}=D_{\alpha}-\left(p_{\eta}-i \bar{\eta}\right) \lambda_{\alpha} \approx 0, \\
\overline{\tilde{\mathcal{D}}}_{\dot{\alpha}} \equiv \overline{\mathcal{D}}_{\dot{\alpha}}-\bar{g} \bar{\lambda}_{\dot{\alpha}}=\bar{D}_{\dot{\alpha}}-\left(\bar{p}_{\eta}+i \eta\right) \bar{\lambda}_{\dot{\alpha}} \approx 0 .
\end{gathered}
$$

All constraints present in the Hamiltonian (A.4) are first class. The only non-vanishing Poisson bracket of them is

$$
\left[\tilde{\mathcal{D}}_{\alpha}, \overline{\tilde{\mathcal{D}}}_{\dot{\alpha}}\right]_{P}=2 i T_{\alpha \dot{\alpha}}
$$

The constraints (A.1) commute with the constraints (2.4), (A.5)-(A.7) but have a nonvanishing Poisson bracket between themselves

$$
[g, \bar{g}]_{P}=2 i
$$

So they are second class. We can introduce the Dirac brackets for them. Then, the constraints (A.5)-(A.7) become (2.3), (2.5) and the variables $\eta, \bar{\eta}$ satisfy commutation relation (2.7). The quantization goes as in Sect. 3 .

Here we outline the alternative Gupta-Bleuler approach to the treatment of the constraints (A.1). When quantizing in this approach, the wave function is assumed to depend on both $\eta$ and $\bar{\eta}$ as the coordinates. Their momenta have the standard realization

$$
\hat{p}_{\eta}=-i \partial / \partial \eta, \quad \hat{\bar{p}}_{\eta}=-i \partial / \partial \bar{\eta}
$$

We impose on the wave function

$$
\tilde{F}^{(q)}(x, \zeta, \bar{\zeta}, y, \bar{y}, \eta, \bar{\eta})
$$

all first class constraints (2.4), (A.5)-(A.7) and half of the second class ones. For definiteness, we take the constraint $\bar{g}$. Thus, the equations on the wave function are

$$
\begin{gathered}
-i\left(\frac{\partial}{\partial \bar{\eta}}+\eta\right) \tilde{F}^{(q)}=0 \\
\left(\partial_{\alpha \dot{\beta}}+i \frac{\partial}{\partial y^{\alpha}} \frac{\partial}{\partial \bar{y}^{\dot{\beta}}}\right) \tilde{F}^{(q)}=0 \\
{\left[D_{\alpha}+\frac{\partial}{\partial y^{\alpha}}\left(\frac{\partial}{\partial \eta}+\bar{\eta}\right)\right] \tilde{F}^{(q)}=0, \quad\left[\bar{D}_{\dot{\alpha}}+\frac{\partial}{\partial \bar{y}^{\dot{\alpha}}}\left(\frac{\partial}{\partial \bar{\eta}}-\eta\right)\right] \tilde{F}^{(q)}=0,} \\
\left(y^{\alpha} \frac{\partial}{\partial y^{\alpha}}-\bar{y}^{\dot{\alpha}} \frac{\partial}{\partial \bar{y}^{\dot{\alpha}}}-\eta \frac{\partial}{\partial \eta}+\bar{\eta} \frac{\partial}{\partial \bar{\eta}}\right) \tilde{F}^{(q)}=q \tilde{F}^{(q)} .
\end{gathered}
$$

Eq. (A.10) is solved by

$$
\tilde{F}^{(q)}(x, \zeta, \bar{\zeta}, y, \bar{y}, \eta, \bar{\eta})=e^{-\eta \bar{\eta}} F^{(q)}(x, \zeta, \bar{\zeta}, y, \bar{y}, \eta)
$$

where the reduced wave function $F^{(q)}$ does not depend on $\bar{\eta}$. Inserting the solution (A.14) in the remaining eqs (A.11)-(A.13), we find that $F^{(q)}$ obeys just eqs. (3.3)-(3.6) which were obtained in Sect. 3. 


\section{Appendix B: Equivalence of the master model and the model with bosonic "supersymmetry"}

In this Appendix we prove the classical equivalence of the master system (2.1) and the HS particle (1.5) with bosonic "supersymmetry". The proof is based on reducing both systems to physical degrees of freedom.

We shall use the light-cone notations

$$
\begin{array}{ccc}
x^{+} \equiv x^{\dot{1} 1}=\frac{x^{0}+x^{3}}{\sqrt{2}}, & x^{-} \equiv x^{\dot{2} 2}=\frac{x^{0}-x^{3}}{\sqrt{2}}, & x^{(t r)} \equiv x^{\dot{2} 1}=\frac{x^{1}+i x^{2}}{\sqrt{2}} \\
P_{+} \equiv P_{1 \dot{1}}=\frac{P_{0}+P_{3}}{\sqrt{2}}, & P_{-} \equiv P_{2 \dot{2}}=\frac{P_{0}-P_{3}}{\sqrt{2}}, & P^{(t r)} \equiv P_{2 \dot{1}}=\frac{P_{1}+i P_{2}}{\sqrt{2}} .
\end{array}
$$

The system (1.5) in physical variables. In the notation (B.1), (B.2) the constraints of the system (1.5) take the following form

$$
\begin{aligned}
& P_{+} P_{-}-\left|P^{(t r)}\right|^{2} \approx 0 \\
& D_{1}=\pi_{1}+i P_{+} \bar{\zeta}^{\dot{1}}+i \bar{P}^{(t r)} \bar{\zeta}^{\dot{2}} \approx 0, \quad \bar{D}_{\dot{1}}=\bar{\pi}_{1}-i P_{+} \zeta^{1}-i \bar{P}^{(t r)} \zeta^{2} \approx 0, \\
& D_{2}=\pi_{2}+i P^{(t r)} \bar{\zeta}^{\dot{1}}+i P_{-} \bar{\zeta}^{\dot{2}} \approx 0, \quad \bar{D}_{\dot{2}}=\bar{\pi}_{\dot{2}}-i P^{(t r)} \zeta^{1}-i P_{-} \zeta^{2} \approx 0 .
\end{aligned}
$$

We choose the light-cone gauge (it is assumed that $P_{+} \neq 0$ )

$$
\begin{gathered}
u^{-} \equiv x^{-}-\tau \approx 0, \\
\zeta^{2} \approx 0, \quad \dot{\zeta}^{\dot{2}} \approx 0 .
\end{gathered}
$$

The constraints (B.5) and the gauge fixing conditions (B.7) eliminate phase variables $\zeta^{2}, \pi_{2}$ and their complex conjugates without affecting the commutation relations for the remaining variables. But the gauge (B.6) involves the proper time. To correctly account for it, we make a canonical transformation from the system with the coordinate $x^{-}$to the system with the coordinate $u^{-}$defined by the relation (B.6). The generating function of this canonical transformation is given by

$$
F=P_{-}^{(u)}\left(x^{-}-\tau\right)+\cdots,
$$

where dots stand for the identical transformation for other variables. This generating function produces the relation (B.6) and yields $P_{-}^{(u)}=P_{-}$. Thus, the conditions (B.6) and (B.3) eliminate $u^{-}$and imply

$$
P_{-}=\frac{1}{P_{+}}\left|P^{(t r)}\right|^{2}
$$

We also should take into account that the canonical transformation depends on the proper time and, therefore, generates the new Hamiltonian (initial Hamiltonian is equal to zero)

$$
H=\frac{\partial F}{\partial \tau}=-P_{-}=-\frac{1}{P_{+}}\left|P^{(t r)}\right|^{2}
$$

As a result, in physical variables the system (1.5) is described by the action

$$
S^{p h}=\int d \tau\left(P_{+} \dot{x}^{+}+\bar{P}^{(t r)} \dot{x}^{(t r)}+P^{(t r)} \dot{\bar{x}}^{(t r)} \pm i(\dot{\bar{\eta}} \eta-\bar{\eta} \dot{\eta}) \mp \frac{1}{\left|P_{+}\right|}\left|P^{(t r)}\right|^{2}\right),
$$


where we introduced $\eta=\sqrt{\left|P_{+}\right|} \zeta^{1}$. In the action (B.11) the upper (lower) sign is chosen for $P_{+}$ positive (negative).

The system (2.1) in physical variables. The first class constraint (2.5) and the gauge-fixing condition for it

$$
\zeta^{\alpha} \approx 0, \quad \bar{\zeta}^{\dot{\alpha}} \approx 0
$$

eliminate the variables $\zeta^{\alpha}, \pi_{\alpha}$ and their complex conjugates without affecting the Poisson brackets for other variables. The remaining constraints (2.3) and (2.4) take the form

$$
\begin{gathered}
\mathcal{N}-c \equiv p^{(\phi)}-2 \eta \bar{\eta}-c \approx 0 \\
P_{+}-\left(r_{1}\right)^{2} \approx 0, \\
P_{-}-\left(r_{2}\right)^{2} \approx 0, \\
P^{(t r)}-r_{1} r_{2} e^{-2 i \psi} \approx 0, \quad \bar{P}^{(t r)}-r_{1} r_{2} e^{2 i \psi} \approx 0,
\end{gathered}
$$

where the variables $r_{1}, r_{2}, \phi, \psi$ are defined by

$$
\lambda_{1}=r_{1} e^{i(\phi+\psi)}, \quad \lambda_{2}=r_{2} e^{i(\phi-\psi)} .
$$

The generating function of the canonical transformation from the variables $\lambda_{\alpha}, y^{\alpha}$ and their complex conjugates to the variables $r_{1}, r_{2}, \phi, \psi$ and conjugate momenta of the latter is given by

$$
F=y^{1} r_{1} e^{i(\phi+\psi)}+y^{2} r_{2} e^{i(\phi-\psi)}+c . c .
$$

From this expression we obtain the relation $p^{(\phi)}=i(y \lambda-\bar{\lambda} \bar{y})$ used in the constraint (B.13).

The constraints (B.13), (B.14), (B.16) together with the gauge-fixing conditions for them,

$$
\phi \approx 0, \quad p_{1}^{(r)} \approx 0, \quad p_{2}^{(r)} \approx 0, \quad p^{(\psi)} \approx 0
$$

completely eliminate the degrees of freedom present in the spinors $\lambda_{\alpha}, y^{\alpha}$ and their complex conjugates.

The gauge-fixing condition for the constraint (B.15) is the light-cone gauge (B.6). As in the previous case we should accomplish the gauge-fixing procedure for this gauge. Note that the condition (B.9) now follows from (B.14)-(B.16). As a result, in physical variables the system (2.1) is described by the action

$$
S_{\text {mast }}^{p h}=\int d \tau\left(P_{+} \dot{x}^{+}+\bar{P}^{(t r)} \dot{x}^{(t r)}+P^{(t r)} \dot{\bar{x}}^{(t r)}+i(\dot{\bar{\eta}} \eta-\bar{\eta} \dot{\eta})-\frac{1}{P_{+}}\left|P^{(t r)}\right|^{2}\right) .
$$

As follows from (B.14), in this case $P_{+}>0$. Thus in the sector with $P_{+}>0$ the actions (B.11) and (B.20) are identical to each other.

\section{References}

[1] E.S. Fradkin and M.A. Vasiliev, Ann. of Phys. 177 (1987) 63; Phys. Lett. B 189 (1987) 89.

[2] M.A. Vasiliev, Fortschr. Phys. 36 (1988) 33; Phys. Lett. B 243 (1990) 378, B 257 (1991) 111. 
[3] E.S. Fradkin and V.Ya. Linetsky, Ann. of Phys. 198 (1990) 252.

[4] E. Sezgin and P. Sundell, JHEP 0109 (2001) 036 [hep-th/0105001].

[5] M.A. Vasiliev, Phys. Rev. D 66 (2002) 0660066 [hep-th/0106149].

[6] M.A. Vasiliev, Higher-Spin Theories and Sp(2M) Invariant Space-Time, the talk presented at the third Sakharov Conference in Physics, Moscow, 26-29 June 2002 [hep-th/0301235].

[7] M.A. Vasiliev, JHEP 0412 (2004) 046 [hep-th/0404124].

[8] D. Sorokin, Introduction to the Classical Theory of Higher Spins, XIX Max Born Symposium "Fundamental Interactions and Twistor-like Methods", Wroclaw, Poland, 28 Sept.-1 Oct. 2004, AIP Conf.Proc. 767(2005)172 [hep-th/0405069].

[9] X. Bekaert, S. Cnockaert, C. Iazeolla and M.A. Vasiliev, Nonlinear Higher Spin Theories in Various Dimensions, Lectures given at Workshop on Higher Spin Gauge Theories, Brussels, Belgium, 12-14 May 2004 [hep-th/0503128].

[10] I. Bandos and J. Lukierski, Mod. Phys. Lett. A 14 (1999) 1257 [hep-th/9811022].

[11] I. Bandos, J. Lukierski and D. Sorokin, Phys. Rev. D 61 (2000) 045002 [hep-th/9904109].

[12] I. A. Bandos, J. A. de Azcárraga, J. M. Izquierdo and J. Lukierski, Phys. Rev. Lett. 86 (2001) 4451 [hep-th/0101113];

I. A. Bandos, J. A. de Azcárraga, J. M. Izquierdo, M. Picon and O. Varela, Phys. Rev. D69 (2004) 105010 [hep-th/0312266].

[13] M. Plyushchay, D. Sorokin and M. Tsulaia, JHEP 0304 (2003) 013 [hep-th/0301067].

[14] I. Bandos, P. Pasti, D. Sorokin and M. Tonin, JHEP 0411 (2004) 023 [hep-th/0407180].

[15] I. Bandos, X. Bekaert, J.A. de Azcárraga, D. Sorokin and M. Tsulaia, JHEP 0505 (2005) 031 [hep-th/0501113].

[16] O.A. Gelfond, E.D. Skvortsov and M.A. Vasiliev, Higher Spin Conformal Currents in Minkowski Space, hep-th/0601106.

[17] S. Fedoruk and J. Lukierski, Phys. Lett. B 632 (2006) 371 [hep-th/0506086]; Higher Spin Particles with Bosonic Counterpart of Supersymmetry, the Proceedings of the Int. Workshop SQS'05, Dubna, July 27-31, 2005, hep-th/0512183.

[18] S. Fedoruk and V.G. Zima, JETP Lett. 61 (1995) 251; Class. Quant. Grav. 16 (1999) 3653 [hep-th/9807192]; J. Kharkov Univ., 585 (2003) 39 [hep-th/0308154].

[19] S. Fedoruk, Twistor Transform for Spinning Particle, XIX Max Born Symposium "Fundamental Interactions and Twistor-like Methods", Wroclaw, Poland, 28 Sept.-1 Oct. 2004, AIP Conference Proceedings, 767(2005)106.

[20] C. Devchand and O. Lechtenfeld, Nucl. Phys. B 516 (1998) 255 [hep-th/9712043]. 
[21] R. Casalbuoni, Nuovo Cimento A 33 (1976) 389;

J.A. de Azcárraga and J. Lukierski, Z. Phys C 30 (1986) 221; Phys. Rev. D 38 (1988) 509.

[22] A.Ferber, Nucl. Phys. B 132 (1978) 55.

[23] Y. Eisenberg and S. Solomon, Nucl. Phys. B 309 (1988) 709;

Y. Eisenberg, Phys. Lett. B 225 (1989) 95.

[24] N. Bengtsson and M. Cederwall, Nucl. Phys. B 302 (1988) 81.

[25] P.K. Townsend, Phys. Lett. B 261 (1991) 65.

[26] S. Fedoruk, E. Ivanov, work in preparation.

[27] A. Galperin, E. Ivanov, V. Ogievetsky and E. Sokatchev, Harmonic Superspace, Cambridge University Press, Cambridge, 2001, 306 p.

[28] A. Galperin, E. Ivanov, S. Kalitzin, V. Ogievetsky and E. Sokatchev, Class. Quant. Grav. 1 (1984) 469.

[29] R. Penrose and M.A.H. MacCallum, Phys. Reports 6C (1972) 241.

[30] P.K. Townsend, Class. Quant. Grav. 8 (1991) 1231.

[31] I. Bars, Lectures on Twistors, hep-th/0601091.

[32] E. Sezgin and E. Sokatchev, Phys. Lett. B 227 (1989) 103.

[33] E. Bergshoeff, M. Blencowe and K. Stelle, Commun. Math. Phys. 128 (1990) 213.

[34] P.S. Howe and P.K. Townsend, Class. Quant. Grav. 7 (1990) 1655.

[35] E. Ivanov, L. Mezincescu and P.K. Townsend, Fuzzy $C P(n \mid m)$ as a quantum superspace, In "Symmetries in Gravity and Field Theory", eds. V. Aldaya, J.M. Cerveró and P. Garciá, Ediciones Universidad de Salamanca, 2004, pp. 385-408, hep-th/0311159.

[36] V. Ogievetsky and E. Sokatchev, Phys. Lett. B 79 (1978) 222.

[37] E. Ivanov and J. Lukierski, Phys. Lett. B 624 (2005) 304 [hep-th/0505216]. 